Investigation of the consistency of magnetic and soft X-ray plasma position measurements on TCV by means of a rapid tomographic inversion algorithm

J. Mlynar, S. Coda, A. Degeling, B.P. Duval,

F. Hofmann, T. Goodman, J.B. Lister, H. Weisen 


\title{
Investigation of the consistency of magnetic and soft X-ray plasma position measurements on TCV by means of a rapid tomographic inversion algorithm
}

\author{
J.Mlynar ${ }^{1}$, S.Coda, A. Degeling, B.P.Duval, F.Hofmann, T.Goodman, \\ J.B.Lister and H.Weisen \\ Centre de Recherches en Physique des Plasmas, \\ Association EURATOM - Confédération Suisse, EPFL, Lausanne, \\ Switzerland \\ 'from January 2003: Institute of Plasma Physics, \\ Czech Academy of Sciences, Prague, Crech Republic
}

a brief version of this report was submitted to Plasma Phys. Contr. Fus.

\begin{abstract}
A streamlined algorithm for tomographic inversion is presented that allows rapid post-discharge reconstruction of the soft X-ray emissivity evolution in the TCV tokamak. Simultaneously it determines the centre of gravity of the emissivity that may serve as a reliable measurement of the plasma position, that is independent of magnetic measurements. Tests of the algorithm were performed using shaped phantom datasets and all past SXR measurements were processed to obtain plasma position statistics. Multiple linear regression is applied in matching the results with the magnetic axis data. Systematic functionalities including magnetic field dependence and drift of the magnetic axis are observed. The joint resolution limit of magnetic and SXR position measurements at $T C V$ is derived from the residual discrepancies and equais $2.2 \mathrm{~mm}$ and $3.1 \mathrm{~mm}$ in the radial and vertical directions, respectively.
\end{abstract}

\section{Introduction}

The TCV (Tokamak à Configuration Variable, $\mathrm{R}=0.88 \mathrm{~m}, \mathrm{a}<0.25 \mathrm{~m}$, $\mathrm{B}_{\Upsilon}<1.54 \mathrm{~T}$ ) with a vessel elongation of 3 is a highly versatile facility for studying the influence of plasma shape on plasma stability and confinement [3]. It is equipped with a flexible ECH heating and current drive system, now totalling 4.5 MW. Most of the ECH power can be steered during the TCV plasma discharge, which allows for highly localised power deposition schemes. Precise and reliable plasma position measurements are thus crucial for both the stability studies and for correctly aiming the ECH system. Contrary to 
other tokamaks, in TCV plasma position and shape can vary significantly even during a single discharge. Both the real time feedback control of the plasma and the post-discharge reconstruction of the plasma magnetic axis and flux surfaces are based on TCV magnetic measurements [1], [2].

At TCV, tomographic inversion is used to reconstruct the plasma emissivity from multiple line integrated measurements from the soft X-ray (SXR) and foil bolometer cameras. Substantial progress in hardware performance motivated the introduction of a speed-optiraised version of the tomographic algorithm. As a result, the SXR and power emissivity tomograms can be reconstructed rapidly after each plasma discharge. In order to respond to the above mentioned needs, position identification by the centre of gravity of the SXR emissivity core was integrated into the rapid tomographic algorithm. The fundamentals of the TCV SXR diagnostics, the applied tomographic inversion and the method of its speed optimisation are described in the following section. The formulas determining the emissivity core position are presented and test runs on phantom data, performed to tune up the algorithm and assess its reliability, are discussed.

Successful performance of the rapid tomography algorithm led to its application to all SXR data available in the TCV database in order to compare statistically the SXR emissivity core position with the stored magnetic axis data. These studies are presented in section 3. Multiple linear regression was applied to quantify the observed systematic deviations. Among others, an influence of toroidal magnetic field on magnetic measurements and a drift of the magnetic axis during discharges were found, clearly demonstrating the potential benefits of field-independent plasma position measurements. Last, but not least, the distribution of remaining discrepancies demonstrate the resolution capabilities of both SXR and magnetic diagnostics.

\section{Rapid algorithm for SXR tomography on TCV}

On TCV, soft X-ray (SXR) tomography analysis is based on data from 10 SXR pinhole cameras, see Fig. 1, each equipped with linear arrays of 20 Centronix photodiodes shielded by $47 \mu \mathrm{m}$ of beryllium foil [4]. The SXR data have been acquired systematically since 1995. General attributes of tomography inversion methods applied in plasma physics are summarised in refs. [5], [6]. On TCV, minimum Fisher regularisation (MFR) on a pixel gxid has been adopted, see [5], [7]. The tomography GUI package tcvxti.m (under MatLab), which was entirely developed and tested in CRPP, was conceived as versatile as possible, with several reconstruction methods included as well as vast support of modelling and simulations. Only limited effort was spent on optimisation of execution speed as overnight batch processing of the data was 
presumed. The time sequence of the resulting tomograms, which represents the evolution of SXR emissivity on the TCV poloidal cross-section, is used to measure the characteristics of plasma poloidal modes via singular value decomposition (SVD) by the GUI package tcvguck. $m$ as described in [5], [8]. This technique proved to be extremely efficient but suffers from long MFR execution times exacerbated by the introduction of a $80 \mathrm{kHz}$ SXR data acquisition system in 1997. A rapid version of the SXR tomography was also required for inter-discharge SXR emissivity analyses and position survey in ECH experiments.

The rapid version of MFR named cattcv.m is now presented. As the first step in the optimisation we streamlined the algorithms of the tcvxtim package so that they run efficiently under MatLab 6.0. Repetitive tasks were replaced by straightforward multidimensional array manipulations, all derived constants were pre-calculated to allow their loading from the disc and repeated access to the TCV database was supressed. This way the total execution time decreased by a half.

Next, we modified the introduction of the geometric matrix $T_{i g}$. In general, inversion methods based on pixels link the signal $f_{2}$ from the $i$-th view to a linear combination of emissivities in the pixels $g_{j}$ :

$$
f_{i} \approx \sum_{3}^{P} T_{i j} g_{3}
$$

where $P$ is total number of pixels and the matrix $T_{i j}$ describes the contribution of the $j$-th pixel emissivity to the signal level in the $i$-th view. Since the plasma is optically thin in the SXR spectral region, the matrix $T_{\imath}$ represents purely geometrical properties of the tomography set-up and may be pre-calculated. In the rapid algorithm we opted for an upload of $T_{i j}$ corresponding to a complete pixel coverage of the TCV vessel cross-section (disregarding plasma size), resulting in significant speed gain at the cost of a larger inversion matrix and some spurious contributions in zero-emissivity regions. The geometrical matrix $T_{i j}$ was pre-calculated on a grid of $15 \times 42$ pixels (size of grid element $\sim 37 \mathrm{~mm}$ ) assuming finite angular widths of the detector views.

The crucial gain in execution speed comes, however, from the adoption of time averaging in the inversion algorithm. In this way, a single inversion matrix $M_{\imath 3}$ can be applied to all timeslices within the analysed time interval:

$$
g_{\jmath \tau}=\sum_{i}^{L} M_{j i} f_{\imath \tau}
$$


where $L$ is the total number of viewing lines and $\tau$ indexes the timeslices. Most present-day high-level programming environments include instructions to evaluate eq.(2) efficiently via a single-line matrix operation.

Let us now examine the time averaging process in more detail. In the MFR method, the inversion matrix $M_{3}$ is given by eq.(26) in [5], which is in our notation

$$
\mathrm{M}=\mathrm{U} \backslash \mathrm{T}
$$

where the backslash stands for left matrix division, i.e. a numerically advantageous transcription of $U^{-1} T$, and the square matrix $U$ reads

$$
U_{i j}=\sum_{l}^{L} T_{i l} T_{l j}+\lambda \sum_{k}^{P} B_{2 k} w_{k} B_{k j}
$$

where $B_{j k}$ is a smoothing matrix describing the influence of the $j$-th pixel on the $k$-th pixel. In the case of MFR the matrix $B_{j k}$ corresponds to firstorder derivation imposed on the pixel grid so that it is, like the matrix $T_{2 y}$, constant for the given setup. Only the regularisation parameter $\lambda$ and the weighting factors $w_{k}$, which describe the global and local smoothing levels, respectively, must be time averaged.

The parameters $\lambda$ and $w_{k}$ are determined in MFR in two nested loops. The inner loop sets the regularisation parameter $\lambda$ so that the residual (misfit) in eq.(1) corresponds to the expected errors in the signals (data errorbars) $\sigma_{i}$. This is done by a $\chi^{2}$ test which in its rapid version includes time averaging in addition to averaging over viewing lines:

$$
\chi^{2}=\frac{1}{S} \frac{1}{L} \sum_{\tau}^{S} \sum_{i}^{L}\left(\frac{f_{\imath \tau}-\sum_{j}^{P} T_{\imath} g_{j \tau}}{\sigma_{\imath}}\right)^{2}
$$

where $S$ stands for the total number of timeslices. In former applications of the pixels method (see e.g. (9]) the smoothing factor $\lambda$ had a role of a free input parameter and $\chi^{2}$ qualified the results. In modern plasma tomography (relying on more preforming hardware) iteration of $\lambda$ targets pre-defined limits of $\chi^{2}$ so that the resulting misfit corresponds to the estimated data error (see e.g. [6]). This simplifies the input choice by passing the smoothing control entirely to the array of errorbars $\sigma_{\imath}$. In practice, the regularisation parameter $\lambda$ is iterated until $\left|\chi^{2}-1\right|<\varepsilon$ is reached or until a limit number of iterations is exceeded; the closest fit is retained in the latter case and a warning message is issued. Notice that the iteration acts quite legitimately and naturally: the higher the errorbars, the higher the smoothing factor $\lambda$ is set in the iteration in order to keep $\chi^{2}$ within pre-defined limits. Furthermore, 
individual terms of the sum in eq. (5) can subsequently help to determine erroneous viewing lines and/or timeslices.

In the external loop, the weighting factors are determined by MFR as shown in $|5|$ but time averaged intensities in pixels $\bar{g}_{3}$ are to be applied:

$$
\begin{aligned}
& w_{j}(n=1)=1 \quad \forall j \\
& w_{j}(n>1)=\frac{\max \left[\bar{g}_{j}(n-1)\right]}{\bar{g}_{j}(n-1)}
\end{aligned}
$$

where $n$ counts the loop cycles. In practice three cycles are applied. The effect of $w_{k}$ is to increase smoothing in low emissivity regions, thus the time averaging process may lead to smoothing distortions when the plasma position changes considerably within the analysed time interval.

Thanks to the above presented modifications the execution time of a typical sequence of $\sim 30$ tomographic reconstructions was reduced to a few percent of its original value, which allows for post-discharge analysis (the CPU needed at TCV IBM machine with 4 power3II $375 \mathrm{MHz}$ processors is now about 10 seconds). In addition, extending the reconstruction of more timeslices does not load the reconstruction algorithm considerably - inverting 130 instead of 30 timeslices increases the execution time by $\sim 10 \%$. This is of great importance particularly for mode analysis by SVD. In this case, moreover, time intervals analysed are invariably short (a few milliseconds), and plasma position changes within them are negligible: consequently, time averaging of $\lambda$ and $w_{j}$ is in fact beneficial in reducing statistical noise. Indeed, the results obtained by the rapid algorithm proved to be comparable to those of the original coding and less sensitive to disturbances in time, see Fig. 2.

A plasma position identification via the centre of gravity of the SXR emissivity was also implemented into the rapid tomography algorithm. Note that from the diagnostics point of view, this introduction of plasma position is disputable. Perhaps more appropriate in SXR data would be the position of the maximum of the first order profile of the SVD of the emissivity. In that case, tomography would have to be run for several time samples around the time coordinate of the position measurement to sweep plasma modes into higher SVD orders. Consequently, time resolution of the position identification would be limited to that of the plasma mode frequency. Moreover, due to insufficient resolution of the pixels grid, a polynomial fit on the first order profile around its maximum would be mandatory. This would further decrease the execution speed of any similar position identification. That is why we finally opted for the simplest and the most reliable position identification which was the centre of gravity of the SXR emissivity. However, regions of low emissivity must be excluded as they are noisy and contain reconstruction 
artefacts. High emissivity levels must also be excluded because they contain largc-scale spatial structures (e.g. plasma modes such as 1,1 island often found at the sawtooth inversion radius) which would lead to incorrect position identification. That is, the resulting plasma position time dependence $\left[r_{\tau}, z_{\tau}\right]$ is determined from the core emissivity evolution:

$$
\left[r_{\tau}, z_{\tau}\right]=\frac{\sum_{\jmath}^{P}\left[r_{3}, z_{j}\right] G_{\jmath \tau}}{\sum_{\jmath}^{P} G_{\jmath \tau}}
$$

where $\left[r_{3}, z_{3}\right]$ are the cylindrical coordinates of the $j$-th pixel's centre and

$$
\begin{array}{lll}
G_{3 \tau}=0 & \text { if } & g_{3 \tau}<l_{\tau} \\
G_{j \tau}=g_{3 \tau}-l_{\tau} & \text { if } \quad l_{\tau} \leq g_{3 \tau} \leq L_{\tau} \\
G_{j \tau}=L_{\tau}-l_{\tau} & \text { if } & g_{j \tau}>L_{\tau}
\end{array}
$$

The upper and lower limits $l_{\tau}$ and $L_{\tau}$ are parametrised as a percentage of the instantaneous maximum emissivity level $\max _{j}\left(g_{3 \tau}\right)$ (see below).

However, the MFR weighted smoothing may cause errors in position identification if the SXR emissivity shifts within the analysed time interval. This adverse MFR feature was demonstrated in model runs performed on a moving phantom function, see Fig. 3. A tomographic reconstruction of SXR data of a real plasma discharge with a narrow profile (\#16110 at $0.6 \mathrm{~s}$ ) was used as a base of the phantom function, its motion was simulated within limits of TCV vessel in both horizontal and vertical directions. A 3\% random Gaussian noise was added to phantom line integrals to model test signal at the detectors. Possible systematic errors in the detector set-up were not taken into account.

Differences between the actual phantom centre of gravity and that of its tomogram were studied as position errors. As can be seen from Fig. 3, the errors due to MFR smoothing asymmetries attained as much as $10 \%$ of the plasma minor radius. As a consequence we decided not to apply weighted smoothing in the position identification. This implies that we apply firstorder linear regularisation tomography method instead of MFR to identify plasma position [5], though in practice we still run MFR and find the centre of gravity within the first cycle of the external loop, eq.(6). The first-order regularisation is less smoothed and thus more noisy at low intensity levels, so that the lower limit $l_{\tau}$ must be set quite high. With these modifications the position errors in test runs decreased to less than $2 \%$ of the plasma minor radius, see Fig. 3.

Once the method was established, test runs on both phantom and real data led us to set the limits in (8) to $l_{\tau}=0.45 \max _{\jmath}\left(g_{j \tau}\right)$ and $L_{\tau}=$ $0.85 \max _{j}\left(g_{j \tau}\right)$. The test runs also indicated that any smoothing method 
to plasma ramp-up and extinction. Eventually we obtained a set of 332520 events (position pairs) of good data quality. These data present an interesting survey of TCV operation in themselves. In Fig. 5 histograms of position of magnetic axis in horizontal and vertical directions are shown. In Fig. 6 histograms of TCV plasma elongations and triangularity are presented.

The initial attempts to compare the magnetic axis position and the SXR emissivity position demonstrated two important features:

1. the statistical error (standard deviation) of the differences between the two position measurements can be very small (less than $3 \mathrm{~mm}$ in certain data subsets) which shows clearly that both methods can identify the plasma position precisely,

2. systematic errors (position shifts) were observed, some of which cannot be attributed to SXR tomography errors

Obviously the challenge is to identify as many causes of systematic errors as possible. We first checked the dependence of the position differences $\Delta r=r_{\text {mag }}-r_{s x r}$ and $\Delta z=z_{\text {mag }}-z_{s x r}$ on the TCV history (see Fig. 7) and discovered limited regions with clear position shifts, some of which correspond to specific experimental configurations. In other words, extension of the position measurements to non-magnetic diagnostics allowed to delimit periods with either distinct or hidden configuration properties that influence the magnetic measurements.

Two of these deserve special mention. First, shift between regions $\mathbf{A}$ and $\mathrm{B}$ in the figure occurs after TCV shutdown in the year 2001, when the grid parameters in the magnetic reconstruction were changed from fixed to discharge-dependent. The shift can be partly ascribed to commutation of plasma drift direction (see below), which was identified at the same time and which may but need not be linked to the modification of the magnetic reconstruction process. Second, region $\mathrm{C}$ fully corresponds to the experimental campaign with inverted toroidal field in the year 1999. In both cases, the shift cannot be an artefact of the rapid SXR tomography, which demonstrates its potential for corrections of slow disturbances in magnetic measurements.

The systematic positive shift in the radial coordinate, on the other hand, can be explained in terms of SXR tomography errors as most of TCV discharges had positive triangularity. As discussed in the previous section, the centre of gravity is shifted inwards from the plasma axis for $\delta>0$ and the tomography smoothing increases this displacement. To evaluate this and other possible effect quantitatively, we applied multiple linear regression (MLR) to 
causes minor systematic shifts in position identification in the direction of asymmetries in the emissivity. To study this effect in detail, a phantom function corresponding to MHD equilibrium for D-shaped elongated plasmas was introduced by applying the Green-Zehrfeld equation [10] to a parabolic emissivity profile. We let the phantom function evolve in time so that its triangularity, elongation and vertical position were scanned independently and then applied the rapid tomography on the whole time sequence. The resulting position error in radial direction as a function of plasma triangularity is shown in Fig. 4, where the dispersion of the result is - approximately to the same degree - due to the phantom position scan and phantom elongation scan. Notice that the difference between the phantom centre of gravity and its axis is also shown (the solid curve). Finally, the dashed line corresponds to linear regression on experimental data, where magnetic (instead of phantom) and SXR tomography position data from different TCV discharges are compared. Its positive offset and slope confirm the expectations but are lower than those predicted by test runs. Detailed presentation of the experimental analyses follows.

\section{Matching the SXR tomography position data to magnetic axis}

The TCV experimental database, which includes broad variations of plasma shapes and positions, offers a unique source for statistical comparison of plasma position data from magnetic and SXR reconstructions. The introduction of rapid SXR tomography with identification of plasma position thus enabled a retrospective study of the benefits of the SXR position measurements face to face standard magnetic reconstructions.

The rapid tomography was run for all SXR data available in the TCV database (discharges \#8500 - \#22885) at the times of previously evaluated magnetic reconstructions. This allowed comparisons of the SXR emissivity position $\left[r_{s x r}, z_{s x r}\right]$ as determined by eq. (7) to the magnetic axis position $\left[r_{\text {mag }}, z_{\text {mag }}\right]$. The latter were calculated after each discharge by the CRPP magnetic reconstruction software package LIUQE [2] using only the TCV magnetic diagnostic measurements [1].

In total, the rapid tomography algorithm successfully reconstructed SXR data from 7023 discharges ( $49 \%$ of the above scope) with a total of 356139 timeslices; in 6257 discharges no SXR data were available and in 1108 discharges the reconstruction did not converge due to poor data or significant radiation from the vessel walls. We subsequently rernoved the results from 698 more discharges which were either too short $(<10$ timeslices, 355 discharges) or noisy (343 discharges) and eliminated timeslices corresponding 
the data as it is available in MatLab statistics toolbox. The routine finds parameters $b_{p}$ and their $95 \%$ confidence intervals (errorbars) that results from the least square fit to the equation

$$
s_{\tau}=D_{\tau b} b_{p}+\varepsilon_{\tau}
$$

where $\tau$ indexes the events, $s_{\tau}$ is a vector of observations, $D_{\tau p}$ matrix of regressors and $\varepsilon_{r}$ vector of remaining discrepancies that are random if all functionalities were covered. In our case, least squares fit between the set of observations $\Delta r$ (or $\Delta z$ ) and several sets of regressors which represent the functionalities are to be determined. We attempted to reflect the interplay of the magnetic field and configuration by the regressors, and eventually applied time $t$, toroidal field parameter $F=R B_{\varphi}$, vertical position parameter $\zeta=I_{p} z_{\text {mag }}$, radial position parameter $\rho=I_{p} r_{\text {mag }}$, plasma elongation $\kappa$ and plasma triangularity $\delta$ in the matrix of regressors. All the regressors were centered around their mean values in order to determine the data offset $b_{0}$ :

$\Delta r_{\tau}=b_{0}+b_{1}\left(t_{\tau}-\bar{t}\right)+b_{2}\left(F_{\tau}-\bar{F}\right)+b_{3}\left(\zeta_{\tau}-\bar{\zeta}\right)+b_{4}\left(\rho_{\tau}-\bar{\rho}\right)+b_{5}\left(\kappa_{\tau}-\bar{\kappa}\right)+b_{6}\left(\delta_{\tau}-\bar{\delta}\right)+\varepsilon_{\tau}$

An identical relation was solved for the second set of observations, $\Delta z_{r}$. Cross-corelation checks on the six regressors were applied to prove that they are lesser than data variance, i.e. that the regressors are statistically independent. The cross-corelation coefficient resulted to be comparable with the data variance only in case of interplay between the two position and/or the two shape parameters, which can be explained in terms of database limitations (e.g. high elongation severely limits the vertical position bandwidth).

Notice that non-proportional (e.g. derivatives) and non-linear functionalities may be at play so that the remaining discrepancies $\varepsilon_{\tau}$ in (10) are not necessarily random. Indeed, a first check of the $\varepsilon_{\tau}$ distribution on the MLR of all 332520 events showed the following:

- the discrepancies have low deviation only after the TCV restart in July 1998 (discharges above \#13765)

- the regression did not fully compensate for the shifts in case of low $I_{p}$ discharges with EC current drive [11] - type E in Fig. 7

- the regression did not compensate the shift between region $\mathbf{A}$ and region $\mathrm{B}$

The regression highlights the deep shift in region $\mathrm{C}$ as a substantial value of the magnetic field coefficient $b_{2}$ in eq.(10) in combination with negative field in the region. It should be noted that $b_{2}$ does vary significantly if 
the region $\mathrm{C}$ is excluded from the regression. Furthermore, a simple plot of dependence of vertical mismatch data on toroidal field intensity clearly confirms this statement, see Fig. 8.

In view of these observations, the final regression is limited to data obtained since 1998 and run separately for regions $\mathbf{A}+\mathbf{C}$ (before 2001 shutdown, i.e. with fixed grid in the magnetic reconstruction) and region $\mathrm{B}$ (discharge-dependent grid since 2001), see Tab. I and Tab. II in the Appendix, respectively. Moreover, the series of low $I_{p}$ discharges with EC current drive has been excluded from the regression, as the interplay of magnetic regressors seems to be different in this case and their statistics is poor. Let us point out the principal results of the regression:

- the analyses give reliable data to within millimeters, that is in the order of a per cent of plasma size

- the modification of magnetic reconstruction in 2001 improved the fit in standard deviations of $\varepsilon_{\tau}$

- a few coefficients reversed their sign due to the modification in 2001, namely vertical position and magnetic field coefficients $b_{2}, b_{3}$ (in both directions) and time coefficient $b_{1}$ (in the vertical direction)

- until 2001, the toroidal field played the principal role in the regression, whereas at present it is the vertical position parameter

Note also the positive offset and positive triangularity coefficient in the radial fit that can be qualitatively explained via the expected tomography shift, see Fig. 3 where the dashed line correspond to the radial fit in groups $\mathrm{A}+\mathrm{C}$ and its errorbars are due to other regression coefficients. However, the results also show that plasma triangularity has an even more important effect in the vertical fit, which cannot be ascribed to the SXR tomography.

Few conclusions can be drawn from the fact that the offset $b_{0}$ and the dependence on vertical plasma position $b_{2}$ are more important in the recent discharges of group B than in group A. This is rather due to the fact that during the SXR tomography commissioning phase the positioning and geometry of the SXR detectors had been adjusted to results of magnetic reconstruction for small circular plasmas [12\}. At that time, the magnetic reconstruction may have been more erroneous than in its present version.

The dependence on the time regressor deserves more attention. It corresponds to a drift in the position difference which can be distinguished in longer discharges even by the naked eye, see Fig. 9. In most TCV discharges the plasma position is supposed to be constant in the plateau phase and is 
fixed by feedback stabilization by the PID (proportional-integral-derivative) controller [13]. While the post-discharge magnetic reconstruction confirms the constant plasma position, the SXR emissivity centre witnesses the drift. The origin of the drift is unclear and can be linked to an electronic drift in the analogue integrators of the TCV magnetic measurements. To further analyse the effect, the mean time derivative of the position mismatch was found for each discharge with least square fit and the results are present cd in histogram form, see Fig. 10. As shown in the figure, in the regions $\mathrm{A}+\mathrm{C}$ the mean value of the vertical drift $v_{z}=-d(\Delta z) / d t$ was found to be $2.4 \mathrm{~mm} . \mathrm{s}^{-1}$ downward, in the region B $1.4 \mathrm{~mm} . \mathrm{s}^{-1}$ upward. The radial drift $v_{r}=-d(\Delta r) / d t$ does not change direction and is considerably reduced, about $0.6 \mathrm{~mm} \cdot \mathrm{s}^{-1}$ outwards. These values qualitatively correspond to regression coefficients $b_{1}$ from Tabs. I, II whereas the exact values differ due to influence of other regressors. Although attempts to quantify functionalities of these drifts resulted in large errors, some of the qualitative observations are worth mentioning:

- the speed of the vertical drift depends on plasma elongation, $d v_{z} / d \kappa>0$ in all regions

- in the region $\mathrm{B}$ only, the vertical drift clearly increases with the vertical position: $d v_{z} / d \zeta>0$

- in all regions, the dependence of $v_{z}$ on plasma triangularity is nonlinear, with a minimum at $\delta \cong 0.3$

- the radial drift $v_{r}$ has a nonlinear dependence on vertical position, with a maximum around $\zeta=0$

Similarly to this drift analysis, the overall efficiency and consistency of the regression (10) was based on statistics of individual discharges. Mean position differences in both the vertical $(\overline{\Delta z})$ and horizontal $(\overline{\Delta r})$ directions were found for all discharges which were used in the above studies. The mean discharge values of the remaining discrepancies $\bar{\varepsilon}_{\tau}$ were also determined; in addition, the standard deviation of the remaining discrepancies $\sigma\left(\varepsilon_{\tau}\right)$ was evaluated for each discharge. The results are shown in histogram form in Fig. 11. While the distributions of $\overline{\Delta z}, \overline{\Delta r}$ are obviously not random, the distributions of both $\bar{\varepsilon}_{\tau}$ and $\sigma\left(\varepsilon_{\tau}\right)$ show characteristics of random noise. Consequently we believe that the mean value of $\sigma\left(\varepsilon_{\tau}\right)$ using all discharges is the best measure of the resolution limit of both magnetic and SXR position measurements. Thus we may conclude that the resolution limit is $3.1 \mathrm{~mm}$ in the vertical direction and $2.2 \mathrm{~mm}$ in the radial direction. It is beyond the scope 
of this work to determine whether one of the diagnostics performs considerably better than the other. However, there are several methods which can further verify the plasma position, let us mention e.g. studies of up-down symmetries in ECH or application of the AXUV tomography (in project). An attempt to back up the position data by the tomographic reconstruction of foil bolometry data [14] failed because of its insufficient spatial resolution.

As the distribution of the input data is quite unbalanced namely in the vertical position, see Fig. 5, we have also tried and run the multiple linear regression on the database with a strongly (10 times) suppressed number of data hits around $z_{\text {mag }}=0.3 \mathrm{~m}$. The results were quite close to the above presented analyses and need not further mention.

The above results were also compared with magnetic data of discharges having a more sophisticated magnetic reconstruction. The TCV database contained 549 discharges with magnetic data correction to Thomson diagnostic results ('LIUQE2') and 51 discharges with a refined iteration limits of the magnetic reconstruction. The statistics of these data are poor but still we may conclude that the observed functionalities do rot differ qualitatively from the above results using the default 'LIUQE1' reconstruction. In future analyses the database could be extended by recalculating the magnetic reconstruction with different parameters (e.g. limited grid on data of groups $A+C)$, however the magnetic reconstruction routine is much more demanding on execution time than the rapid SXR tomography. Benefits of such an effort are also mitigated by uncertainties in the long-term stability of PID plasma controller.

\section{Conclusions}

The presented TCV rapid tomography algorithm allows for immediate post-discharge emissivity reconstruction. This rapidity is attributable to time averaging of the inversion matrix which was demonstrated to provide reliable results, enabling the rapid algorithm to be routinely used for the tomographic analysis. Detailed studies of selected emissivity tomograms should still be checked using the previous step-by-step algorithm and also verified by a different tomographic method.

The position of the SXR emissivity core is identified by the rapid algorithm, allowing systematic studies of its relation to TCV magnetic axis. The statistical analyses of the resulting database with 332520 points show satisfactory resolution of both diagnostics $(3.1 \mathrm{~mm}$ in vertical direction and $2.2 \mathrm{~mm}$ in radial direction) and, at the same time, it reveals minor inaccuracies of the magnetic diagnostics including its sensitivity to toroidal field intensity and a positional drift during plasma discharges. 
These studies persuaded us of the advantages of field independent measurements for correcting and enhancing the magnetic reconstructions. This conclusion applies not only to TCV, but to magnetically confined plasmas in general (see e.g. [15]), including the reactor projects like ITER. At the same time, progress in computer speed and capacity allow the initiation of work on programmable real-time tomogxaphy systems with feedback to the low frequency part of plasma control systems [16]. The feedback can be mandatory, for example, in plasma steady state operation to reinitialize the integrators drifting due to radiation induced electromotive force in the cable insulator [17]. Note that the soft X-ray diagnostics suitable for tomography are tractable even under reactor conditions if, for instance, nnultiwire imaging chambers are used [18].

It is important to note that the emission centre of gravity is different from the magnetic axis position when the field configuration is asymmetric in the poloidal plane. Similarly, in the high ion temperature reactor plasmas the SXR emissivity core may be offset from the centre. In automated applications, all such systematic shifts need to be software compensated, which should not present a major hindrance. Self-taught neural style system is an option, keeping in mind that ITER-size machines with applicable low frequency delay of several seconds allow for computationally intensive processes. Consequently, we believe that SXR tomography techniques still offer important opportunities for further developments.

\section{Acknowledgements:}

The authors are thankful to all present and past colleagues in CRPP whose work allowed us to carry out the analyses. Our special thanks go to the TCV control and data acquisition group led by Xavier Llobet and to Christian Ingesson from JET-EFDA for his valuable comments.

A brief version of this report was submitted to Plasma Physics and Cont.rolled Fusion in September 2002.

This work was partly supported by the Swiss National Science Foundation. 


\section{References}

11] Moret, J.-M., Bühlmann, F., Fasel, D. et al., Rev.Sci. Instrum. 69 (1998) 2333

[2] Hofmann, F., Tonetti, G., Nucl. Fusion 28 (1988) 1871

[3] Weisen, H. et al, Nucl. Fusion 41 (2001) 1459-1472 and the TCV website: http://crppwww.epfl.ch/tcv/

[4] Anton. M. et al., Rev. Sci. Instrum. 66 (1995) 3762

[5] Anton, M. et al., Plasma Phys. Control. Fusion 38 (1996) 1849

[6] Ingesson, L.C. et al., Nucl. Fusion 38 (1998) 1675

[7] Anton, M. et al., CRPP EPFL Rep., INT 189/96 (1996)

[8] Furno, I. et al., doctoral thesis, CRPP EPFL Rep., LRP 703/01 (2001)

[9] Williamson, J.H., Culham Rep., CLM-R 210 (1982)

[10] Green, B.J., Zehrfeld, H.P., Nucl. Fusion 17 (1977) 1133

[11] Coda S. et al., Plasma Phys. Control. Fusion 42 (2000) B311

[12] Anton, M., private communication

[13] Lister J.B. et al, CRPP EPFL Rep., LRP 518/95 (1996)

[14] Ch. Schlatter, J. Mlynar, CRPP EPFL Rep., INT 205/02 (2002)

[15] Borrás, M.C., Granetz, R.S., Plasma Phys. Control. Fusion 38 (1996) 289

[16] Mlynar J., Duval B.P., Horacek J., Lister J.B, poster SOFT 2002, Helsinky, submitted to Fusion Engineering and Design

[17] Van Nieuwenhove, R., Verneeren, L., poster SOFT 2002, Helsinky, submitted to Fusion Engineering and Design

[18] Sushkov A. et al., Proc. 29th EPS Conference on Plasma Phys. and Contr. Fusion, Montreux (2002) ECA Vol 26B, P-4.118 


\section{APPENDIX}

Results of multiple linear regression applied on differences between magnetic axis and SXR emission centre of gravity according to the set of eq.(9). The errorbars of parameters $b_{2}$ correspond to $95 \%$ confidence interval of the least squares fit. The quantity $\xi_{2}$ is the standard deviation of individual addends in the same set of eq. (9) so that it may be used as an indicator of the regressor significance in the resulting fit.

Table 1: multiple linear regression, group $\mathrm{A}+\mathrm{C}$ TCV discharges \#13765 - \#20315 (July 1998- April 2001) in total 158446 events

\begin{tabular}{|c|c|c|c|c|c|c|c|}
\hline & & \multicolumn{2}{|c|}{ set of observations $\rightarrow$} & \multicolumn{2}{|c|}{$\Delta z$ (vertical fit) } & \multicolumn{2}{|c|}{$\Delta r$ (radial fit) } \\
\hline 1 & regressor & unit U & mean value & $\mathrm{b}_{i}[\mathrm{~mm} / \mathrm{U}]$ & $\xi_{i}[\mathrm{~mm}]$ & $\mathrm{b}_{2}[\mathrm{~mm} / \mathrm{U}]$ & $\overline{\xi_{\imath}|\mathrm{mm}|}$ \\
\hline 0 & offset. & 1 & - & $2.11 \pm 0.02$ & - & $8.35 \pm 0.02$ & - \\
\hline 1 & time $t$ & $\mathrm{~s}$ & 0.661 & $2.00 \pm 0.06$ & 0.8 & $-0.41 \pm 0.04$ & 0.2 \\
\hline 2 & toroidal field $R B_{\varphi}$ & T.m & 0.973 & $7.31 \pm \overline{ \pm 0.03}$ & 5.3 & $2.89 \pm 0.02$ & $2 . \overline{1}$ \\
\hline 3 & vertical pos. $\overline{I_{p} z}$ & MA.m & 0.057 & \pm 0.9 & 1.7 & \pm 0.6 & 0.6 \\
\hline 4 & radial pos. $I_{p} r$ & MA.m & 0.891 & \pm 0.5 & 0.0 & \pm 0.3 & 0.8 \\
\hline 5 & elongation $\kappa$ & 1 & 1.59 & \pm 0.2 & 0.9 & $2.7 \pm 0.1$ & $0 . \overline{7}$ \\
\hline 6 & triangularity $\delta$ & 1 & 0.278 & \pm 0.3 & 1.0 & $6.4 \pm 0.2$ & $0 . \overline{7}$ \\
\hline
\end{tabular}

standard deviation of remaining discrepancies $\varepsilon_{\tau}: 4.8 \mathrm{~mm}$ in vertical fit, $3.2 \mathrm{~mm}$ in horizontal fit

Table 2: multiple linear regression, group B TCV discharges \#20382 - \#22885 (September 2001 - June 2002) in total 42123 events

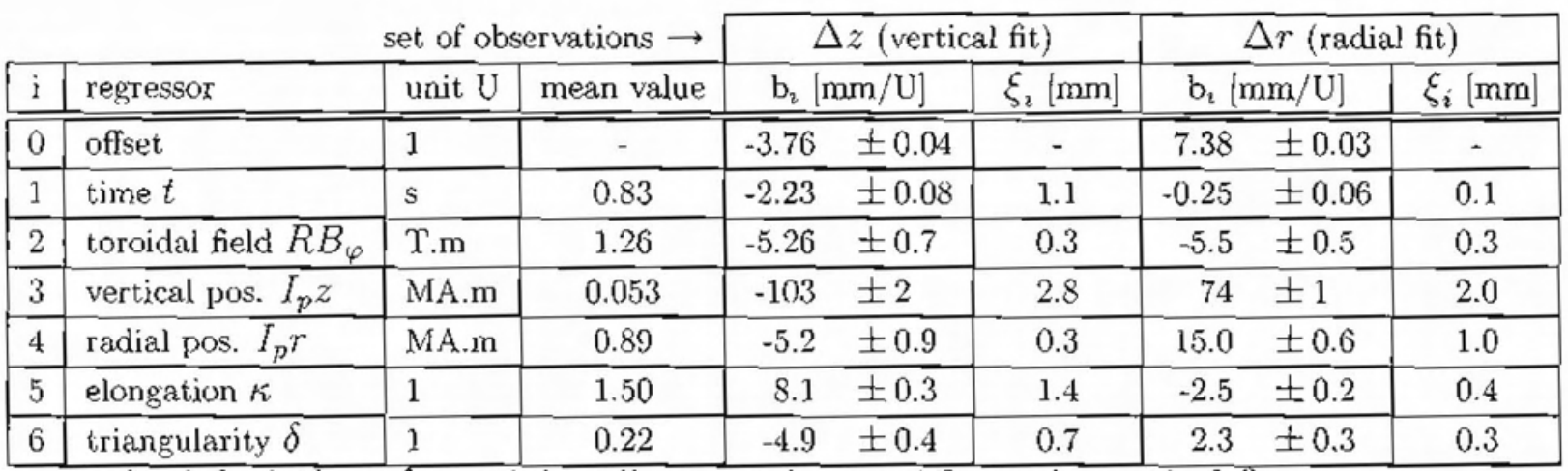

standard deviation of remaining discrepancies $\varepsilon_{\tau}: 4.0 \mathrm{~mm}$ in vertical fit, 


\section{Figure captions:}

Fig. 1: Setup of diagnostics for soft X-ray tomography in a TCV poloidal cross-section setup (left) and in the projection space where each viewing line correspond to a dot and the upper and bottom lines limit the TCV vessel (right). The small square in the edge of the left figure corresponds to a default pixel size in the rapid tomography ( $15 \times 42$ pixels cover the poloidal cross section completely).

Fig. 2: Example of singular value decomposition (SVD) results of SXR emission profiles (discharge \#15963, time interval $0.8010 \mathrm{~s}-0.8013 \mathrm{~s}$ ) as determined by original step-by-step tomography on pixel grid limited to the plasma incidence (left) and by the rapid tomography with the pixel grid covering the whole TCV vessel (right). Both methods give qualitatively identical results ( $\mathrm{m}=2$ poloidal mode). However, the rapid tomography sets lower intensity to the mode due to relatively higher smoathing of the emissivity fluctuations in time. Advantageously, application of a single reconstruction matrix in the rapid tomography smoothes directly the time dependence (chronos) as well as - indirectly - the space resolution (topos).

Fig. 3: Test studies of tomography position measurements performance; difference between phantom centre of gravity and reconstructed centre of gravity is $\Delta r=r_{p h}-r_{s x r}$ in radial and $\Delta z=z_{p h}-z_{s x r}$ in vertical directions. SXR emissivity of a plasma with low poloidal surface (discharge \#16110 at $0.6 \mathrm{~s})$ was used as a phantom function whose motion across the TCV chamber in both vertical $\left(-0.2 \mathrm{~m} \leq z_{p h} \leq 0.2 \mathrm{~m}\right)$ and radial $\left(-0.79 \mathrm{~m} \leq r_{p h} \leq 0.94 \mathrm{~m}\right)$ directions was simulated. As a consequence of plasma motion, the first order regularisation (with standard deviation $2 \mathrm{~mm}$ ) gives considerably better results than minimum Fisher regularisation (standard deviation $10 \mathrm{~mm}$ ) due to asymmetric smoothing in the latter method. Phantom radial asymmetry leads to systematic error in radial direction, see also Fig. 4.

Fig. 4: Radial position systematic errors $\Delta r=r_{p h}-r_{s x r}$ as a function of plasma triangularity (Green-Zehrfeld phantom function). Phantom triangularity, elongation and vertical position were scanned within $-0.7 \leq \delta \leq 0.7$, $1 \leq \kappa \leq 2$ and $-0.2 \mathrm{~m} \leq z_{p h} \leq 0.2 \mathrm{~m}$ while its radial position was kept fixed. The dots corresponds to the difference between the phantom axis and the reconstructed centre of gravity; their radial dispersion is due to the position and elongation scan. The full line shows the centre of gravity of the phantom function as a function of its triangularity. The dashed line presents experimental results according to coefficients $b_{0}$ and $b_{6}$ in Tab $I$ in the Appendix, 
$\Delta r=r_{\text {mag }}-r_{s x r}$. The errorbars correspond to the amplitude of elongation and position dependence.

Fig. 5: Statistics of magnetic axis position according to the TCV database, discharges \#8500-\#22885 (July 1998 - June 2002).

Fig. 6: Statistics of plasma elongation and triangularity according to the TCV database, discharges \#8500-\#22885 (July 1998 - June 2002).

Fig. 7: Differences in magnetic and SXR position measurements in vertical (top) and radial (bottom) directions as a function of TCV history. The following regions (some of them overlapping) are highlighted: $\mathbf{A}$ are mainly discharges before year 2001, B since 2001, C discharges with reversed magnetic field, D early TCV discharges positioned near the bottom of the vessel $(\zeta<0), \mathrm{E}$ EC current driven discharges with low $I_{p}$. The displacement in the radial direction is a consequence of plasma D-shaping, see Fig. 4.

The figure is constituted by data regrouped into consecutive subsets which contained 1000 events each. The $x$-axis indexes the subsets and the gxeyscale corresponds to the distribution of position differences in each subset.

Fig. 8: Dependence of the vertical magnetic and SXR position measurements mismatch on toroidal field amplitude. Each point correspond to an event from the database regions $\mathbf{A}$ and $\mathbf{C}$. This plot clearly explains the deep shift in region $\mathrm{C}$ in Fig. 7. The dashed line slope corresponds to $b_{2}$ value from Table I in the appendix but the offset differs by $5 \mathrm{~mm}$ which is probably due to elimination of other regressors in the $2 \mathrm{D}$ functionality plot.

Fig. 9: Two series of long TCV discharges. Individual discharges are separated by vertical lines. In the discharges with constant magnetic axis position the downward plasma drift (before TCV shutdown in 2001) or the upward plasma drift (after the shutdown) are clearly visible. In case of ECCD the effect vanishes, and in case of the vertical plasma position scan it is compensated by the positional functionality of the mismatch.

Fig. 10: Histograms of vertical and radial drift velocities. Vertical and radial drift velocities were determined by least square fit on differences in magnetic and SXR position measurements in individual TCV discharges. Notice that in the vertical direction there is a substantial difference between region $\mathbf{A}+\mathbf{C}$ (before TCV shutdown in 2001) and region $\mathbf{B}$ (after the shutdown). In radial direction, the mean time derivative is also convincingly non-zero but there is no distinct difference between different regions, see also Table I and Table II in the Appendix. As shown in the figure, in the regions 
$\mathrm{A}+\mathrm{C}$ the mean value of the vertical drift $v_{z}=-d(\Delta z) / d t$ was found to be $2.4 \mathrm{~mm} . \mathrm{s}^{-1}$ downward, in the region B $1.4 \mathrm{~mm} . \mathrm{s}^{-1}$ upward. The radial drift $v_{r}=-d(\Delta r) / d t$ does not change direction and is considerably reduced, about $0.6 \mathrm{~mm} . \mathrm{s}^{-1}$ outwards.

Fig. 11: Results of the regressions in vertical (top) and radial (bottom) directions: distribution of discharge mean values of position differences (left), distribution of discharge mean value of remaining discrepancies $\vec{\varepsilon}_{\tau}$ (middle), distribution of standard deviations of remaining discrepancies per discharge $\sigma\left(\varepsilon_{\tau}\right)$ (right). Mean value of $\sigma\left(\varepsilon_{\tau}\right)$ is shown as a vertical line, $\overline{\sigma\left(\varepsilon_{\tau z}\right)}=3.1 \mathrm{~mm}$ in the vertical direction and $\overline{\sigma\left(\varepsilon_{\tau \tau}\right)}=2.2 \mathrm{~mm}$ in the radial direction. 

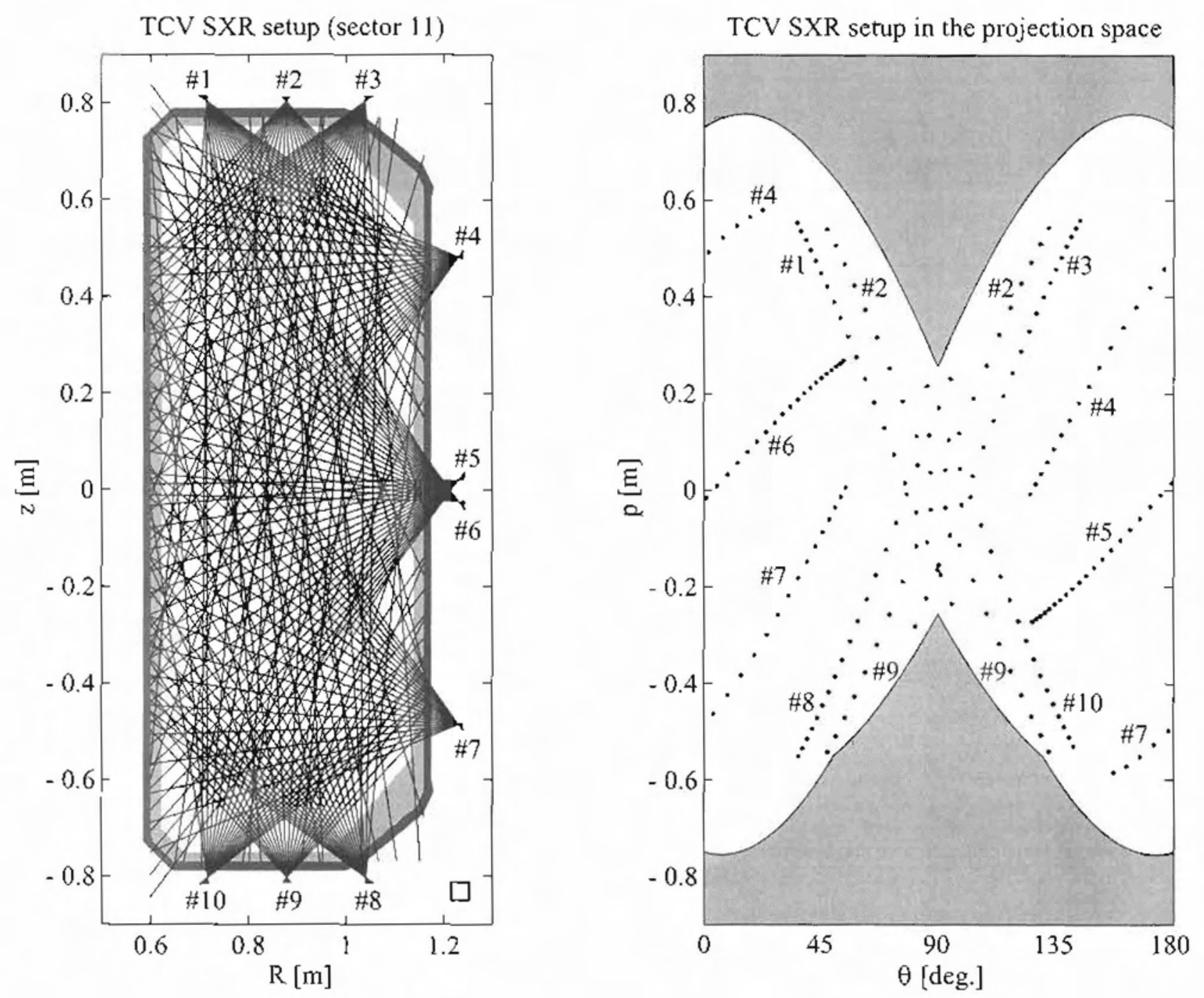

Fig. 1 


\section{TCV discharge \#15963}

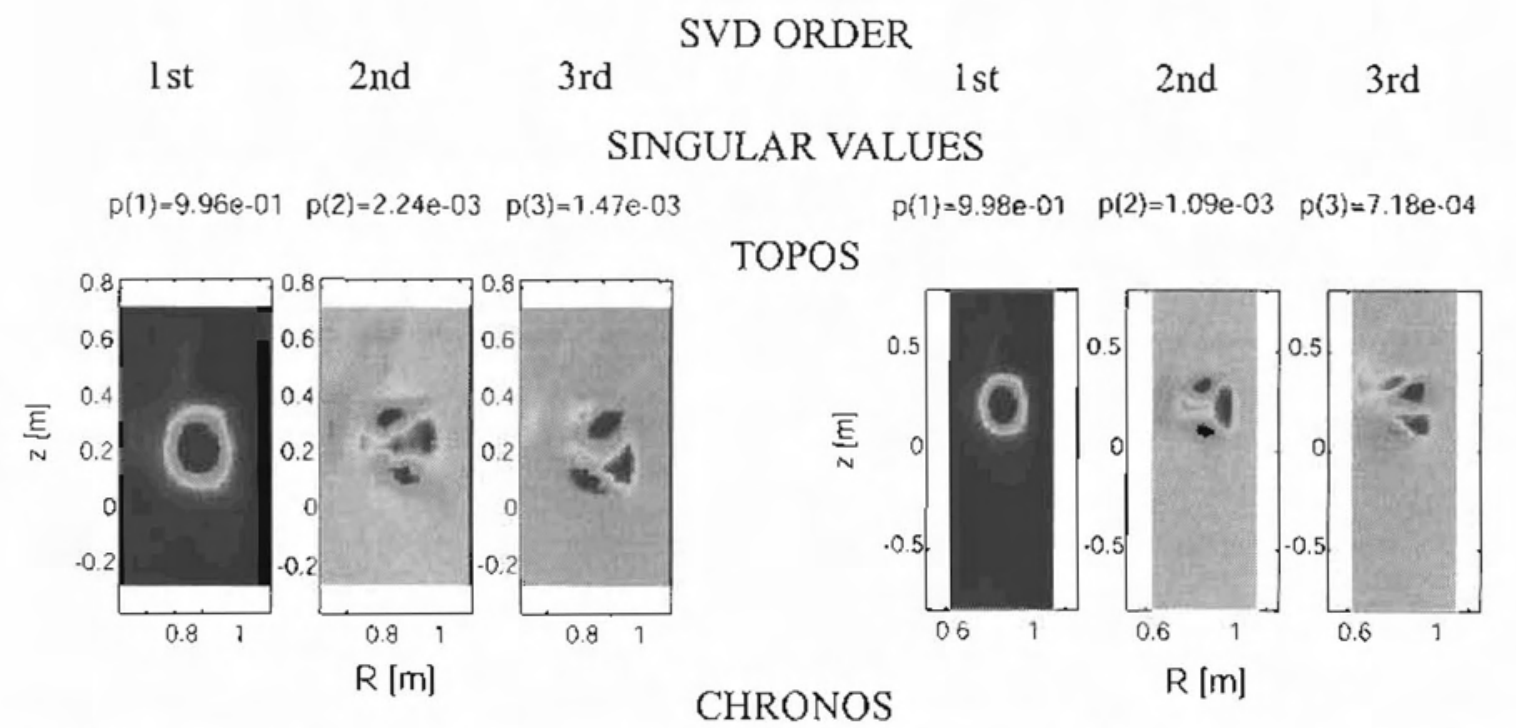

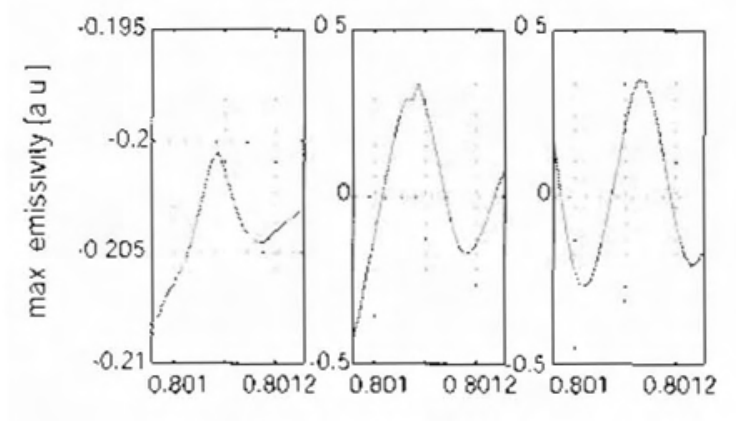

I [s]

STANDARD TOMOGRAPHY

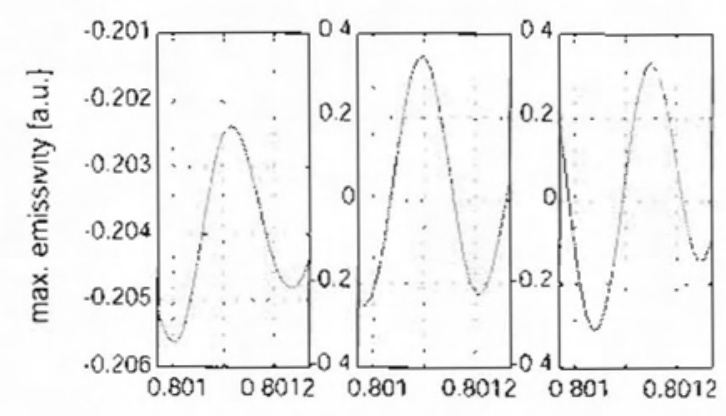

! [s]

RAPID TOMOGRAPHY

Fig. 2 


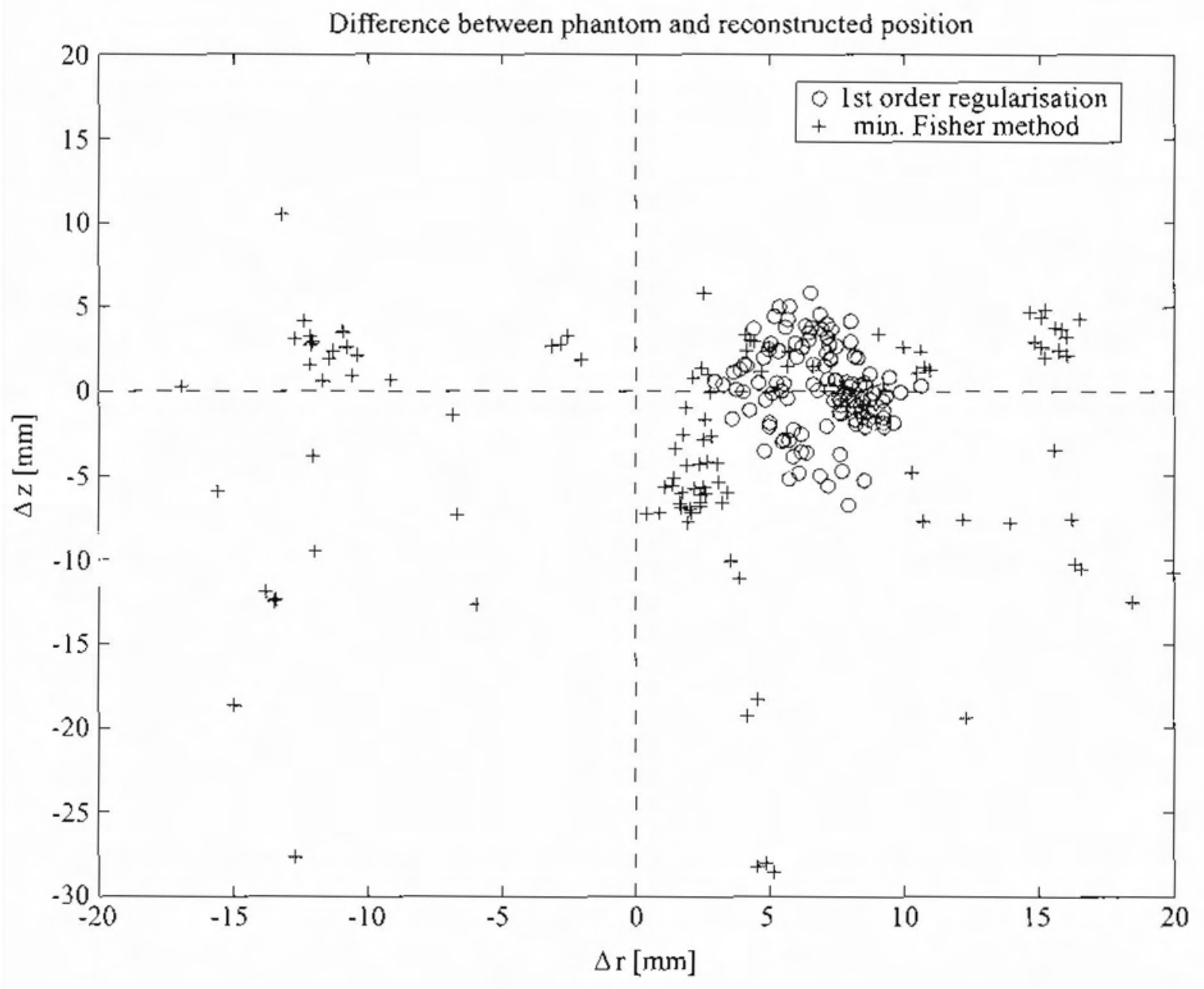

Fig. 3 


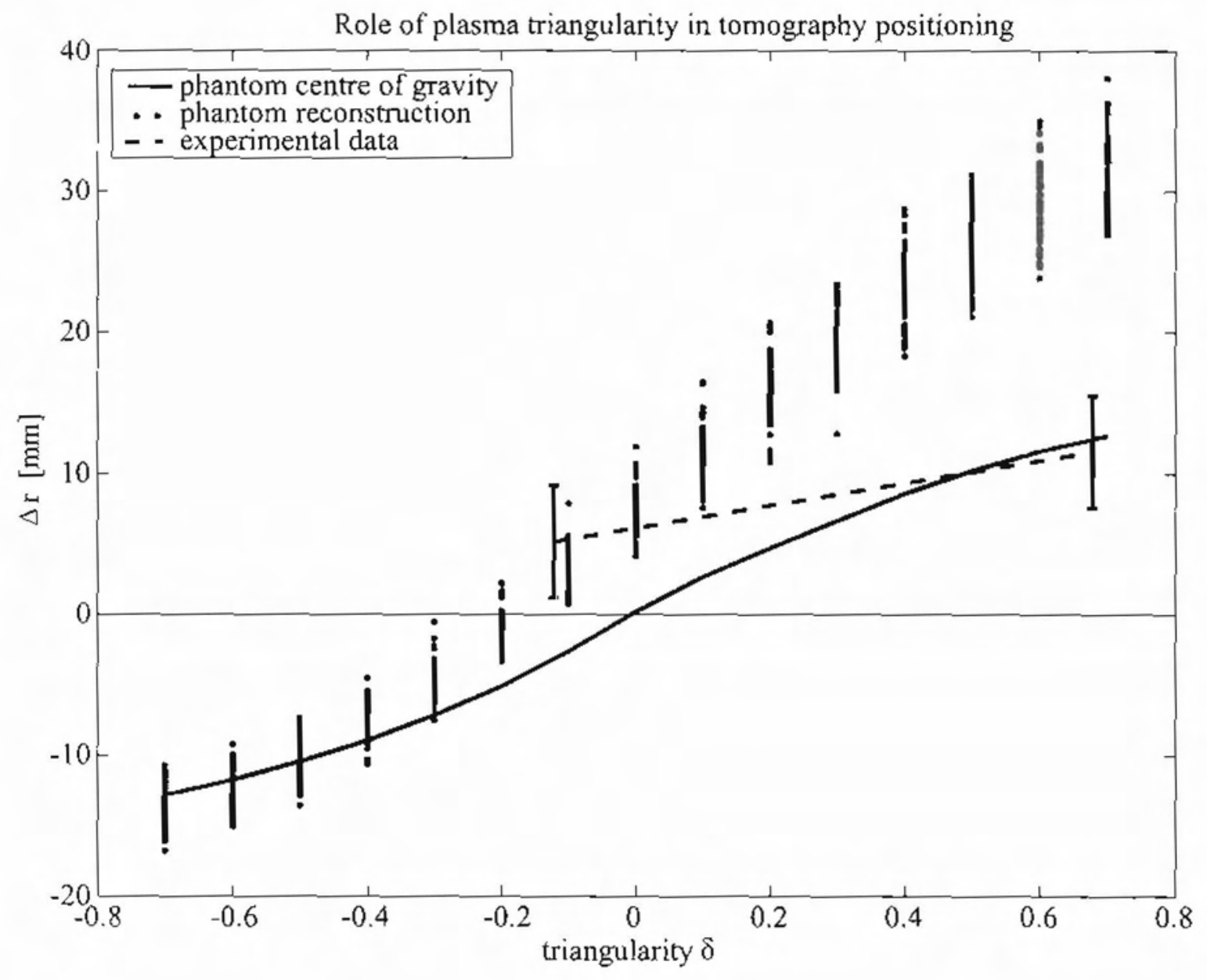

Fig. 4 

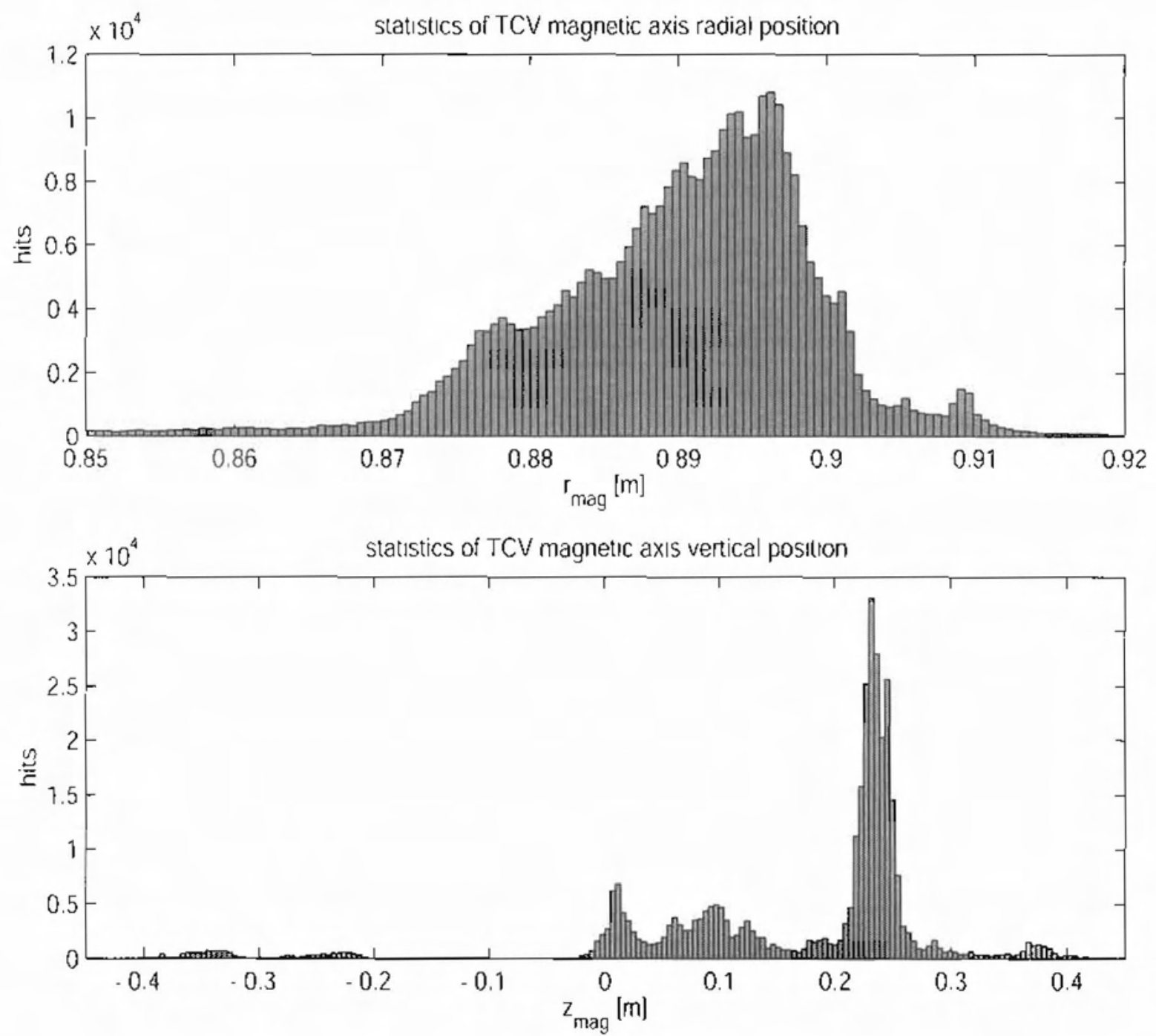

Fig. 5 

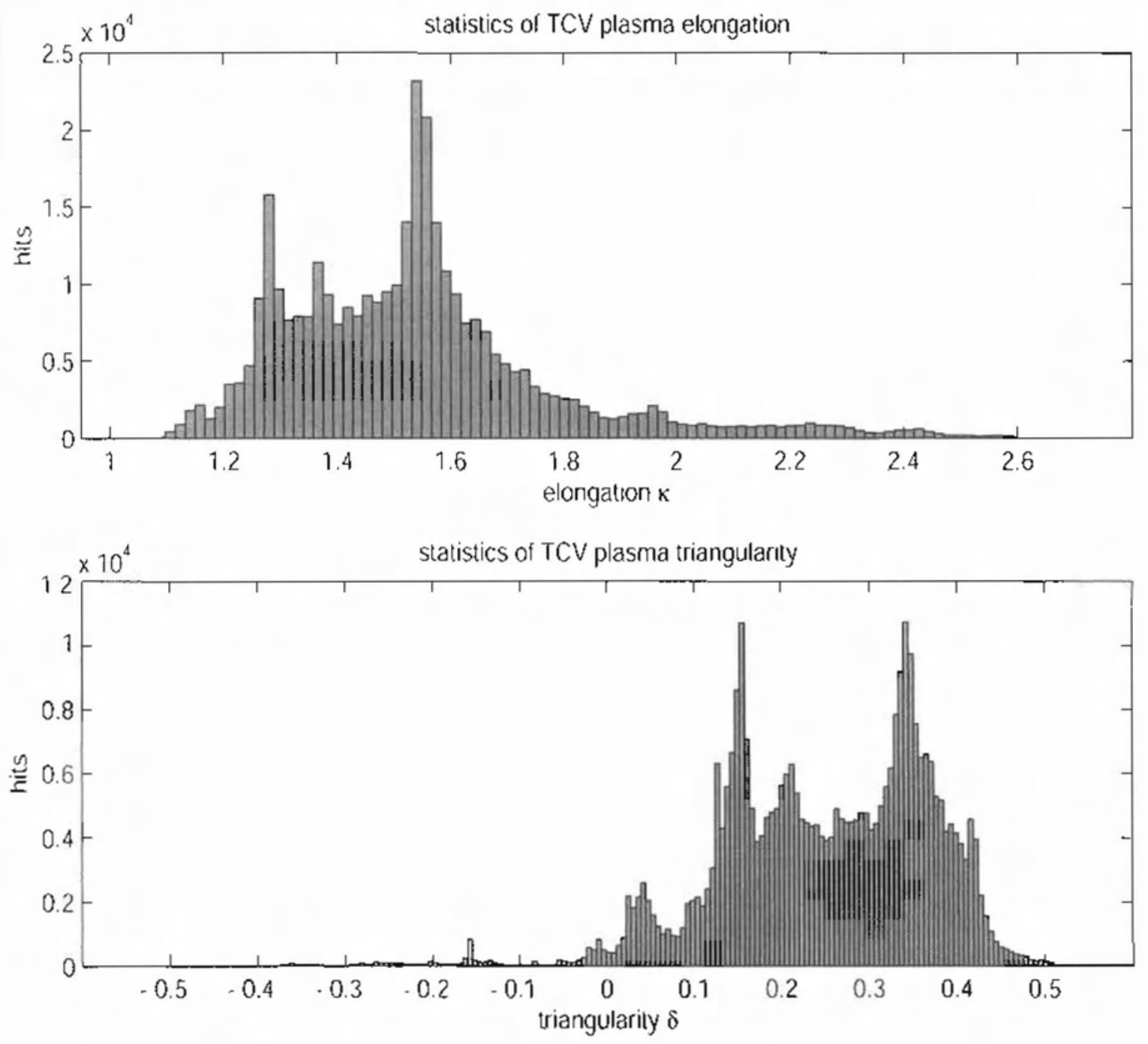

Fig. 6 

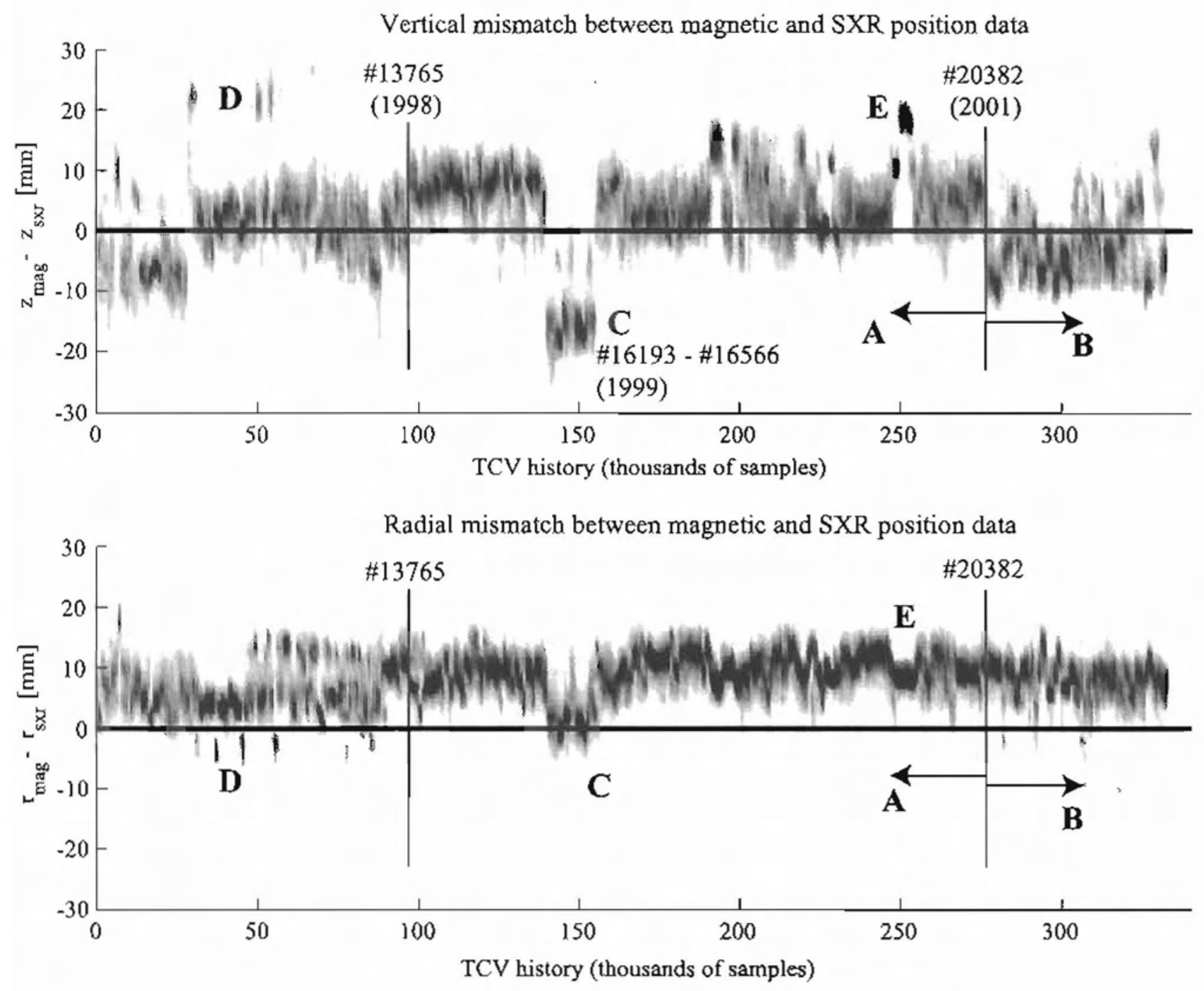

Fig. 7 


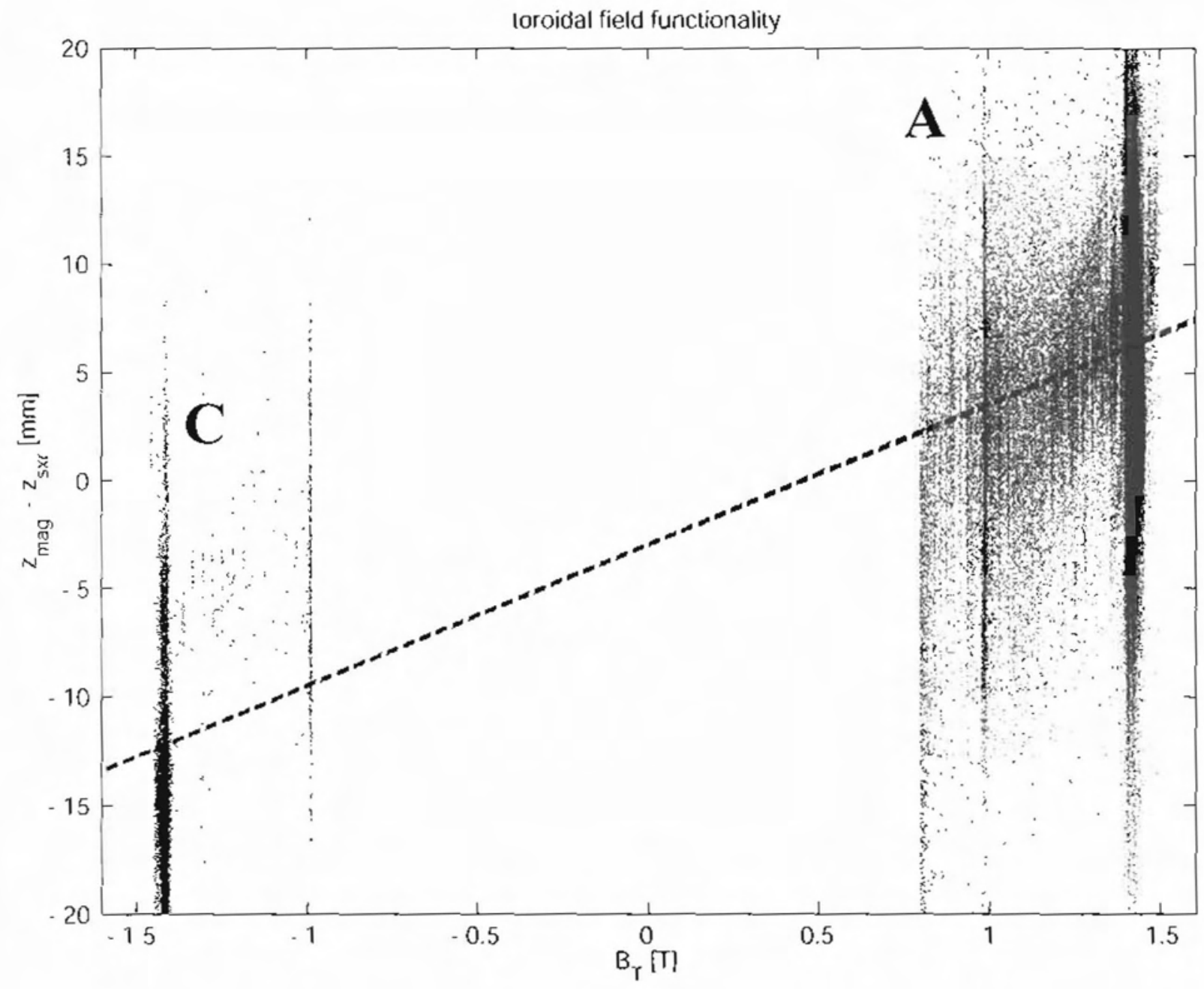

Fig. 8 
Example of drift in vertical plasma position, before...
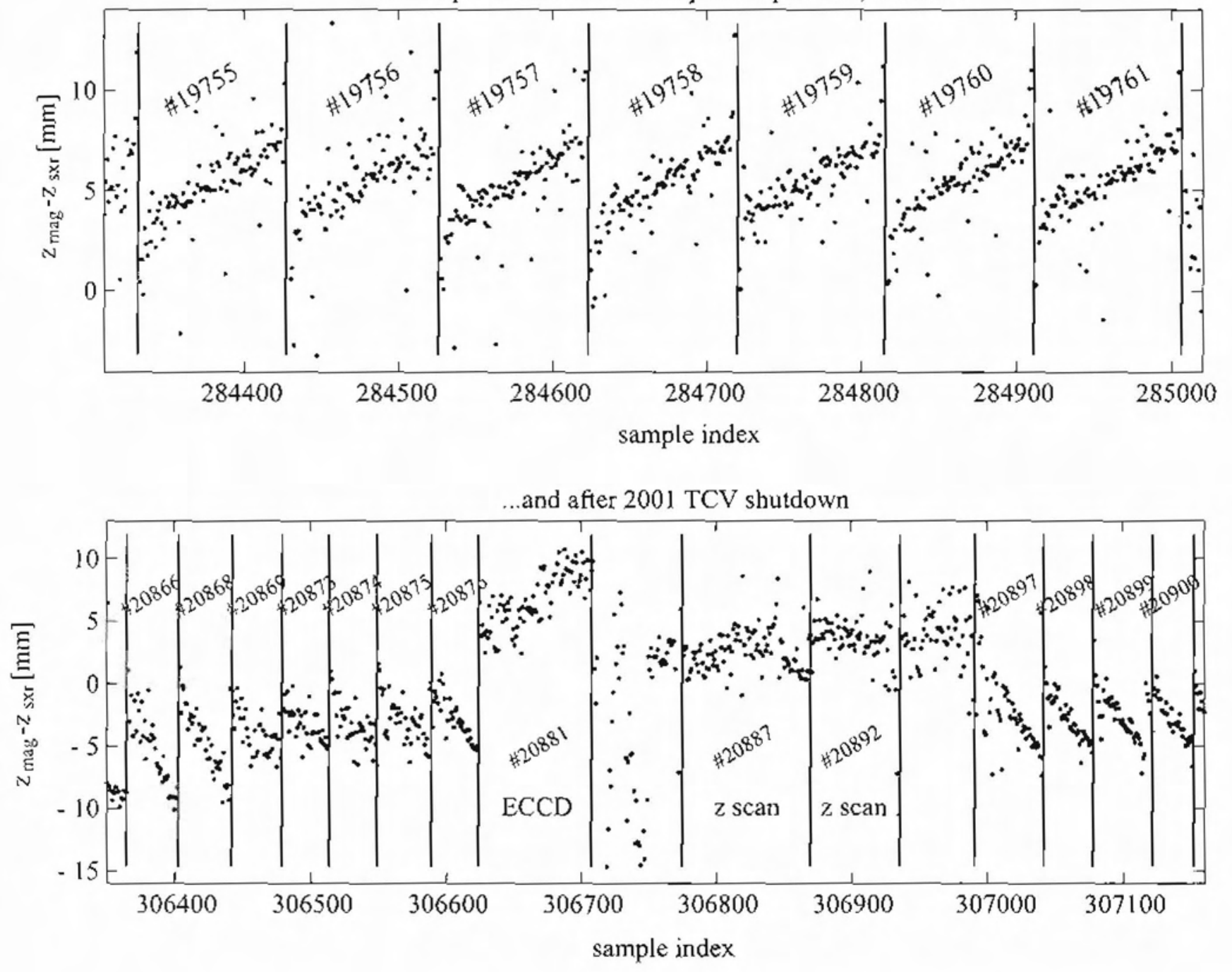

Fig. 9 
Time derivative of the vertical mismatch, before \#20340
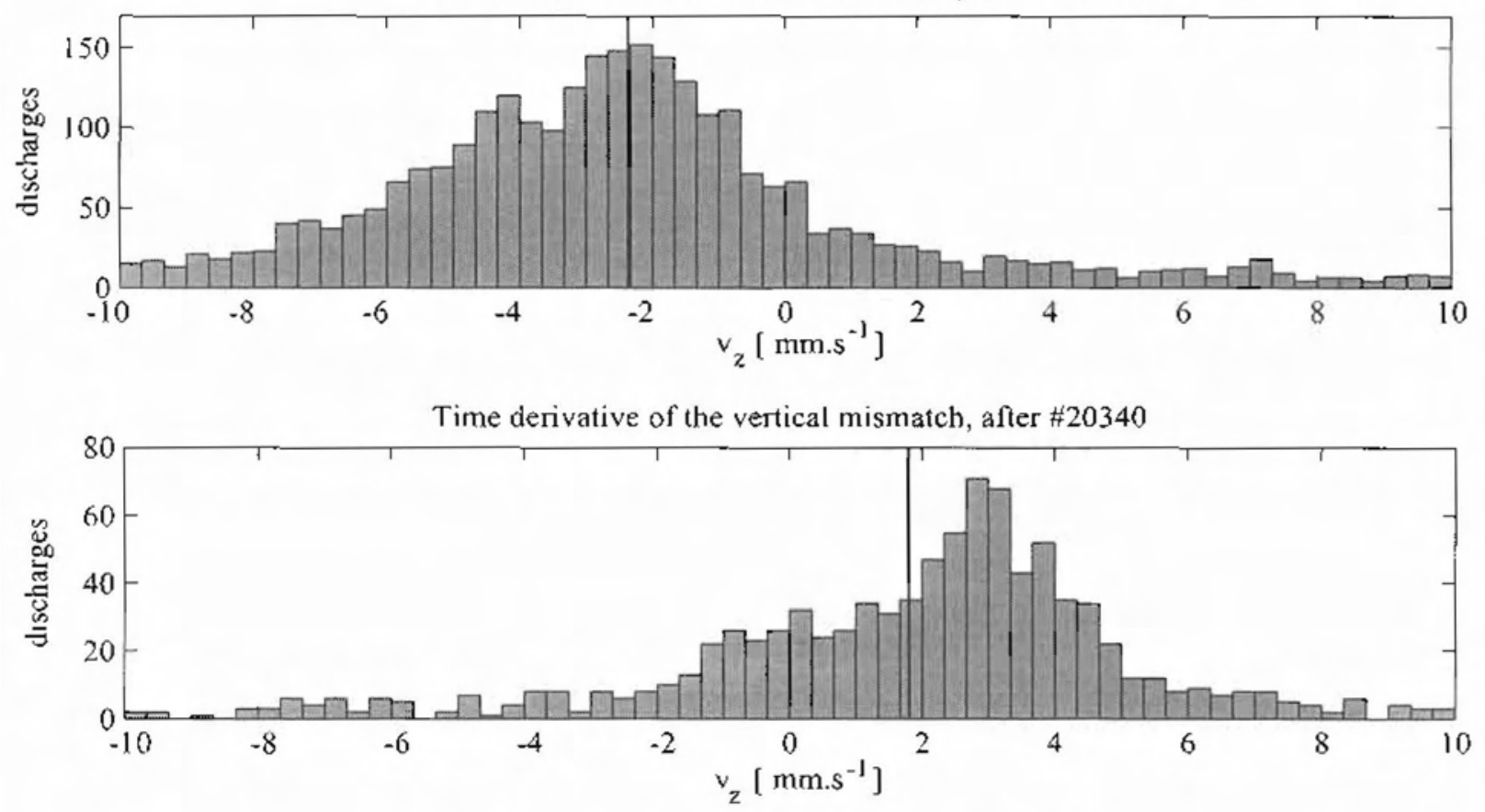

Time derivative of the radial mismatch, all shots

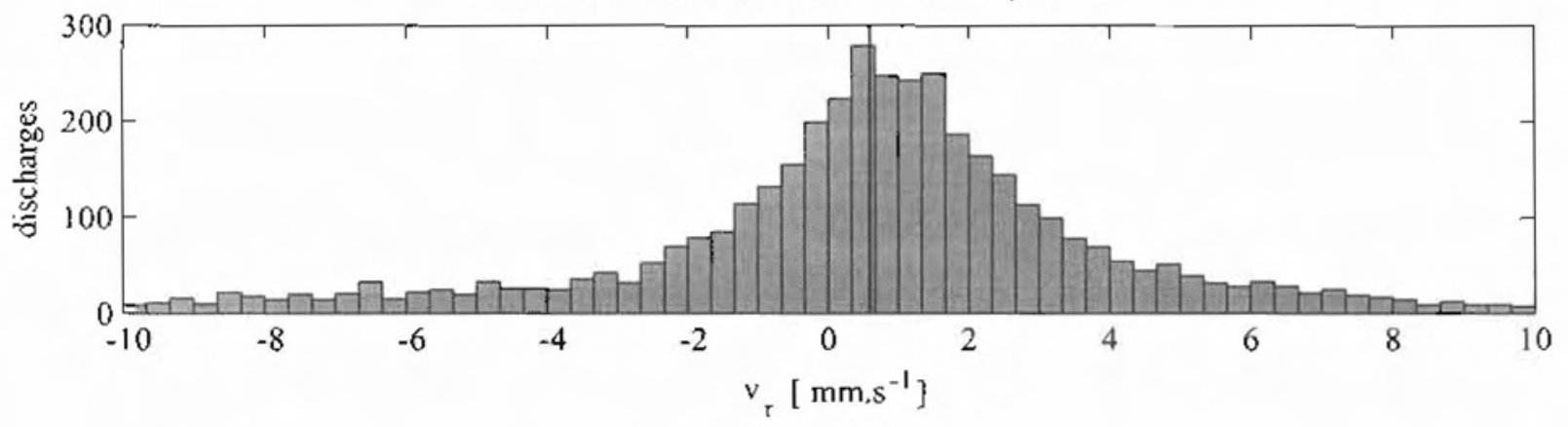

Fig. 10 

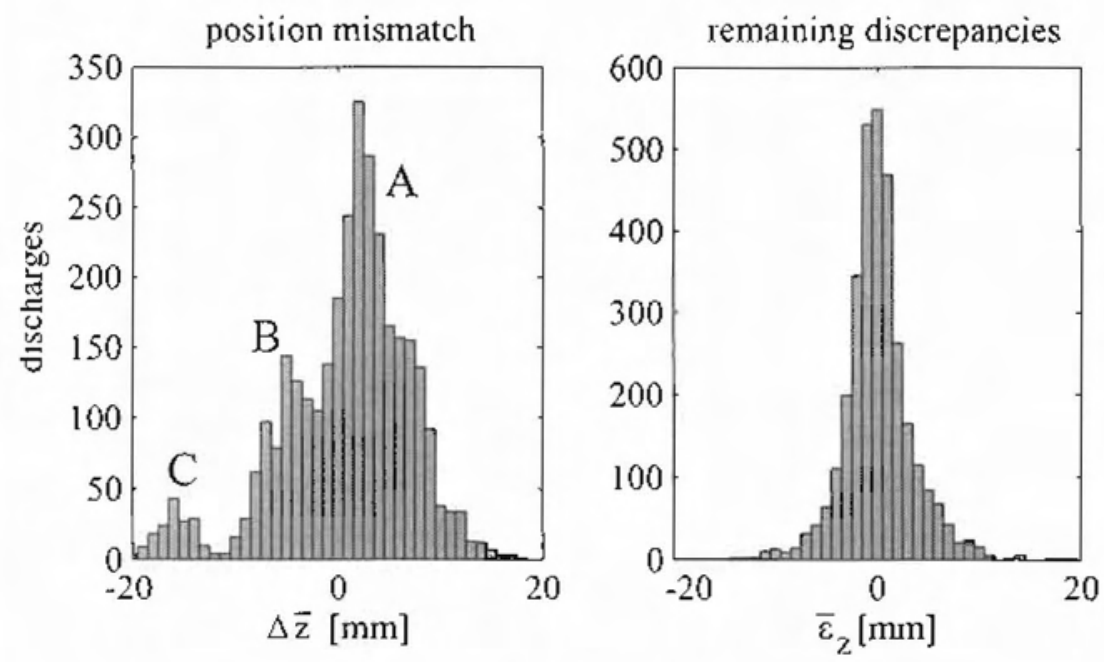

standard deviation of discrepancies
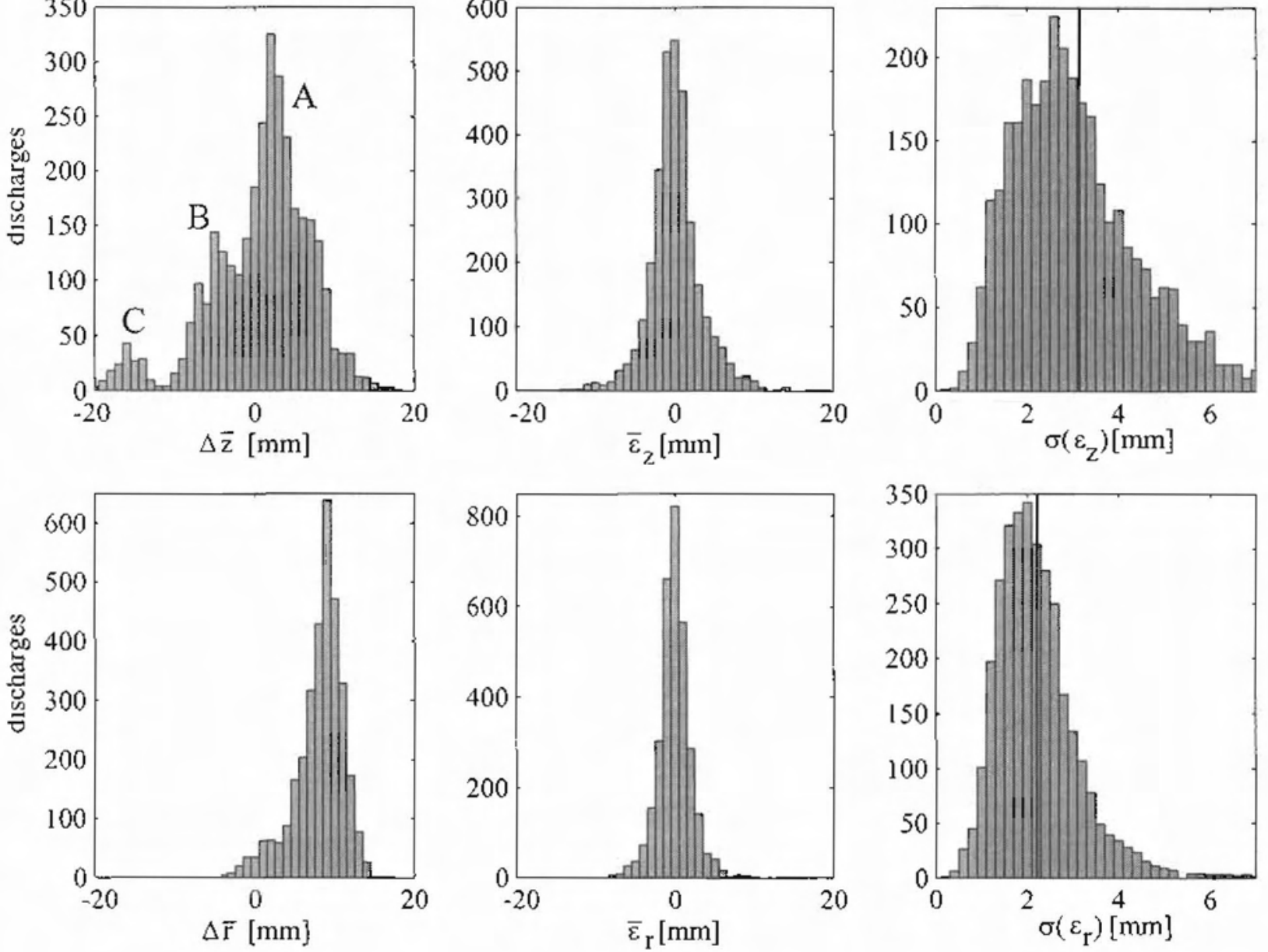

Fig. 11 